\title{
S-1, an oral fluoropyrimidine, enhances radiation response of DLD-1/FU human colon cancer xenografts resistant to 5-FU
}

\author{
EIKO NAKATA ${ }^{1}$, MASAKAZU FUKUSHIMA $^{2}$, YOSHIHIRO TAKAI ${ }^{3}$, KENJI NEMOTO $^{4}$, YOSHIHIRO OGAWA ${ }^{1}$, \\ TAKUMA NOMIYA ${ }^{4}$, YASUHIRO NAKAMURA ${ }^{5}$, LUKA MILAS ${ }^{6}$ and SHOGO YAMADA ${ }^{4}$
}

\begin{abstract}
${ }^{1}$ Tohoku University Biomedical Engineering Research Organaization, 2-1 Seiryocho Aobaku Sendai-City, Miyagi 980-8575; ${ }^{2}$ Pharmacokinetics Research Laboratory, Taiho Pharmaceutical Co., Ltd., 224-2 Ebisuno, Hiraishi, Kawauchi-cho, Tokushima 771-01; ${ }^{3}$ Department of Radiological Sciences, Tohoku University School of Medicine; 2-1 Seiryocho Aobaku Sendai-City, Miyagi 980-8575; Departments of ${ }^{4}$ Radiation Oncology, ${ }^{5}$ Anatomic Pathology, Tohoku University Graduate School of Medicine, 2-1 Seiryocho Aobaku Sendai-City, Miyagi 980-8575; ${ }^{6}$ Department of Radiation Oncology, University of Texas MD Anderson Cancer Center, 1515 Holcombe Blvd., Houston, TX 77030, USA
\end{abstract}

Received February 21, 2006; Accepted April 25, 2006

\begin{abstract}
S-1, a novel oral fluoropyrimidine, is increasingly used for the treatment of human cancer including gastrointestinal carcinomas. Using the 5-FU resistant DLD-1/FU human colon cancer cell xenografts, the present study investigated whether S-1 enhances the therapeutic efficacy of radiation and if so what are the underlying mechanisms. Nude mice bearing tumor xenografts were treated with radiation, $\mathrm{S}-1$, or both. Tumor growth delay was the treatments' endpoint. To determine whether S-1 enhances intrinsic cell radiosensitivity, we performed clonogenic cell survival assay. Also we assessed the expression of thymidylate synthase (TS) using immunohistochemistry assay. While S-1 or 5 Gy were only slightly effective as single agents in delaying tumor growth, the combined treatment was highly effective. Clonogenic cell survival showed that $\mathrm{S}-1$ strongly enhanced cell radiosensitivity. Immunohistochemistry showed that the expression of TS was down-regulated in tumors treated by S-1 plus radiation. Combined $\mathrm{S}-1$ plus radiation treatment resulted in a synergistic effect in the therapy of 5-FU resistant human colon carcinoma xenografts $(\mathrm{EF}=2.06)$. The effect could be attributed to the ability of S-1 to increase cell radiosensitivity $(\mathrm{EF}=1.9)$ and to the down-regulation of TS involved in cellular processes leading to radio- and (or) chemo-resistance.
\end{abstract}

\section{Introduction}

Concurrent chemoradiotherapy, i.e. administering chemotherapeutic agents during the course of radiotherapy, has

Correspondence to: Dr Shogo Yamada, Department of Radiation Oncology, Tohoku University Graduate School of Medicine, 1-1 Seiryocho, Aobaku, Sendai-City, Miyagi 980-8574, Japan

E-mail: shogo-y@rad.med.tohoku.ac.jp

Key words: S-1, radiation, colon cancer become a common strategic practice in the therapy of advanced cancer. There exists a solid biological rationale for combining cytotoxic drugs with radiotherapy (1). Because of their systemic cytotoxic action chemotherapeutic drugs reduce the number of cancer cells in the tumor undergoing radiotherapy and act against tumor deposits outside the radiation field. In addition, these drugs may sensitize tumor cells to the cytotoxic effects of radiation by reducing or eliminating intrinsic cellular radioresistance, repair of sublethal and potentially lethal radiation damage, cell cycle related radio-resistance or tumor hypoxia. Also, the drugs can improve tumor radioresponse by counteracting the rapid regeneration of tumor cells during radiotherapy. The improvements in the treatment outcome after concurrent chemotherapy have been observed in terms of increased local tumor control, patient survival and organ preservation rates (2-6). However, concurrent chemoradiotherapy is limited in its application because it is usually associated with a considerable increased normal tissue toxicity, and in spite of achieved advance in treatment outcome the cure rates in the majority of solid tumors still remain poor (2-5). Thus, there is considerable room for improvement of the combined treatment strategies.

This progress in chemoradiotherapy has been achieved mainly by using standard chemotherapeutic agents, which have traditionally been selected for combined treatment based primarily on their known clinical activity in certain disease sites (2-6). 5-Fluorouracil (5-FU) is one of the standard chemotherapeutic drugs commonly prescribed for the treatment of gastrointestinal (GI) tract and head and neck malignancies either alone or in combination with other agents including radiotherapy $(2,3,6.7)$. 5 -FU is a pyrimidine analog that interferes with both DNA and RNA synthesis. Its major metabolite, fluorodeoxyuridine monophosphate, inhibits the formation of thymidine by blocking thymidylate synthase. Another metabolite of 5-FU, fluorouridine monophosphate, becomes incorporated into RNA and affects RNA synthesis. Combined with radiation, 5-FU can cause either additive or supra-additive effects (8). Interaction with 
irradiation occurs mainly when the drug is administered within 1 day before irradiation and several hours after irradiation; however, the potentiation of radiation response seems to be greatest when 5-FU is present in cultured cells for $\sim 24 \mathrm{~h}$ after irradiation. The exact mechanisms of enhanced cell radiation response are not clear, but they are likely associated with cell cycle redistribution induced by the drug. Clinically, the antitumor efficacy of 5-FU is limited by its toxicity to normal tissues mainly GI toxicity and myelotoxicity, and to its rapid catabolism in the body minimizing its antitumor efficacy $(6,7,9)$.

With respect to 5-FU, a novel fluoropyrimidine derivative, designated S-1, was generated consisting of tegafur (FT), 5chloro-2, 4-dihydrooxypyridine (CDHP) and potassium oxonate (Oxo) in a molar ratio of 1:0.4:1 (10). FT is a prodrug of 5-FU (11). CDHP and Oxo possess no antitumor activity but modulate certain activities of 5-FU. CDHP competitively inhibits dihydropyrimidine dehydrogenase, which degrades 5-FU, resulting in an increased and prolonged retention of 5-FU in the blood (12). Oxo competitively inhibits pyrimidine phosphoribosyltransferase, which converts 5-FU to 5-fluorouridine-5'-monophosphate (13), and after peroral administration it is distributed mainly within the GI tract leading to reduction in 5-FU-induced GI toxicity (13). S-1 exerts a strong antitumor activity in a variety of rodent tumors and human tumor xenografts $(10,14,15)$. In clinical trials, S-1 has shown considerable antitumor efficacy against a number of common cancers in humans including gastric $(16,17)$, colorectal (18), breast (19), head and neck (20) and lung cancer (21).

Because of its potent antitumor efficacy and good tolerability, S-1 represents a good candidate for combining it with radiotherapy. However, limited information is available on the treatment efficacy when this drug is combined with radiotherapy. Recently, Harada et al (22) reported that S-1 increases the in vivo radioresponse of tumor xenografts derived from oral cancer cells, and that its active component, 5-FU, has the ability to sensitize the in vitro radioresponse of these cells. Using human colorectal carcinoma cell xenografts, sensitive or resistant to 5-FU, the present study investigated whether S-1 enhances cellular radiosensitivity and improves the antitumor efficacy of tumor radiotherapy.

\section{Materials and methods}

Mice and tumors. Female BALB/C-nu/nu nude mice, purchased from CLEA Japan (Tokyo, Japan) were 5 to 6 weeks old at the beginning of the experiments and were housed five per cage. The mice were maintained in a specific pathogen-free barrier. Animals used in this study were maintained in facilities approved by the Tohoku university animal facility. Tumor xenografts were derived from the human colorectal carcinoma cell line DLD-1, which is sensitive to 5-FU, and DLD-1/FU, which is resistant to 5-FU. Both cell lines were obtained from the Taiho Pharmaceutical, Co. Ltd (Tokyo, Japan). The 5-FU resistant DLD-1/FU cell line was originally derived from the DLD-1 cell line by continuous in vitro exposure of DLD-1 cells to increasing concentrations of 5-FU through a number of successive passages, as described earlier (23). Source tumors were produced by s.c. injection of $10^{7}$ cells into the back of 4-5weeks-old mice. Tumor cell suspensions were prepared from DLD-1/P or DLD-1/FU cells grown as monolayers in vitro. When the source tumors grew to $200-300 \mathrm{~mm}^{3}$ they were excised and cut into $\sim 2 \mathrm{~mm}^{3}$ fragments, which were then implanted into the right hind leg of BALB/C-nu/nu mice to generate solitary tumors for the experiments.

Local tumor irradiation. Mice bearing $80-100 \mathrm{~mm}^{3}$ size tumors in the right hind leg were locally irradiated with a single dose of $5 \mathrm{~Gy}$. A small animal X-ray generator, with a dose rate of $0.72 \mathrm{~Gy} / \mathrm{min}$ was used. Unanesthetized mice were immobilized on a jig during irradiation. The irradiation was delivered locally to the tumor whereas the remaining body of the mouse was shielded. When $\mathrm{S}-1$ and radiation were combined, S-1 was given orally $1 \mathrm{~h}$ before the start of irradiation and continued once daily for 14 days.

$S-1$ and 5-FU. S-1 is a chemotherapeutic agent prepared by simultaneous mixing of FT, CDHP and Oxo in a molar ratio of 1:0.4:1 and dissolved in $0.5 \%$ hydroxypropylmethylcellulose (HPMC). FT, CDHP and Oxo are products of the Taiho Pharmaceutical Co. Ltd. S-1, at a dose of $8 \mathrm{mg} / \mathrm{kg}$, was given to mice orally in a volume of $0.1 \mathrm{ml} / 10 \mathrm{~g}$ body weight daily for 14 consecutive days. The dose of S-1 was expressed as the dose of FT. 5-FU was purchased from Sigma (St. Louis, MO, USA).

Clonogenic cell survival determination. Tumor cells in culture were exposed to 5 -FU at $\mathrm{IC}_{50}$ doses (inhibition of cell growth 50), $5.8 \mu \mathrm{M}$ for DLD-1 cells or $350 \mu \mathrm{M}$ for DLD$1 / \mathrm{FU}$ cells, for 2 days. Then the cells were irradiated with graded doses $(2,4$, or $6 \mathrm{~Gy})$ of X-rays $0.72 \mathrm{~Gy} / \mathrm{min}$. The cells were assayed for colony-forming ability by replating them in specified numbers into $100 \mathrm{~mm}$ dishes in a drug-free medium. After 8 days of DLD-1 cell incubation and 14 days of DLD-1/FU cell incubation, the cells were stained with $0.5 \%$ crystal violet in absolute ethanol, and colonies with more than 50 cells were counted. Radiation survival curves were plotted after normalizing for the cytotoxicity induced by $5-\mathrm{FU}$ alone. Clonogenic survival curves were constructed from three independent experiments by fitting the average survival levels using least squares regression by the linear quadratic model (24).

Tumor growth delay. Tumor growth delay was the endpoint of treatments (vehicle for the control group, S-1, radiation or both), which were initiated when tumors grew to $80-100 \mathrm{~mm}^{3}$ in volume $\left[1 / 2 \times\right.$ (the major axis) $\mathrm{x}$ (the minor axis) $\left.{ }^{2}\right]$. To obtain tumor growth curves, two mutually orthogonal tumor diameters were measured with a vernier caliper at 2-3 day intervals and the volumes were calculated. Regression and regrowth of tumors were followed until tumor reached about $1000 \mathrm{~mm}^{3}$, at which time the mice were sacrificed by cervical dislocation. Tumor growth delay was expressed as the time in days for tumors treated with radiation or S-1 to grow to 4 times their pretreatment volume minus the time in days for untreated tumors to reach the same volume. This is termed as the absolute growth delay (AGD). The effect of the combined TS-1 plus radiation treatment was expressed as 
A

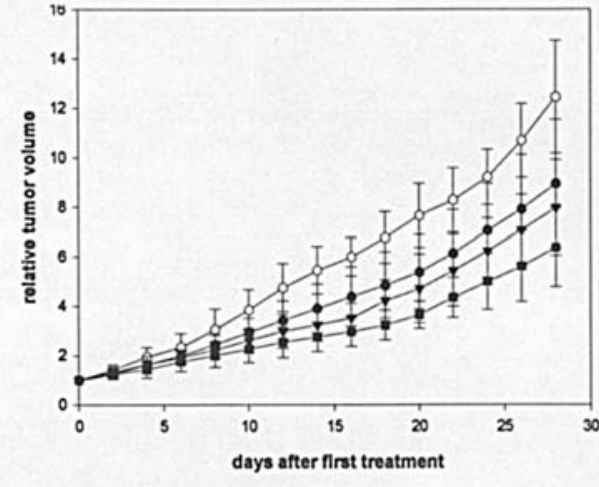

B

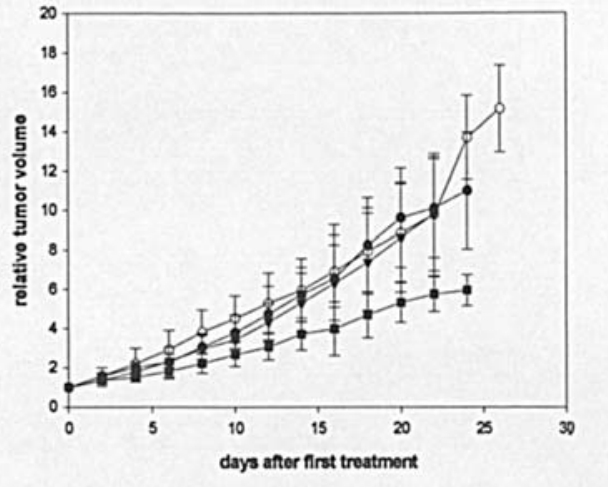

Figure 1. (A) Effect of S-1 on the growth of DLD-1 (sensitive to 5-FU) tumor xenograft. $\bigcirc$, no treatment; $\mathbf{\nabla}, \mathrm{S}-18 \mathrm{mg} / \mathrm{kg} ; \bullet \bullet, \mathrm{radiation} 5 \mathrm{~Gy}$; $\mathbf{\bullet}, \mathrm{S}-18 \mathrm{mg} / \mathrm{kg}$ and radiation $5 \mathrm{~Gy}$. Each data point represents the mean volume of 6-9 tumors. Bars, SE. (B) Effect of S-1 on the growth of DLD-1/FU (resistant to 5-FU) tumor xenograft. o, no treatment; $\mathbf{v}, \mathrm{S}-18 \mathrm{mg} / \mathrm{kg}$; $\bullet$, radiation $5 \mathrm{~Gy}$; $\mathbf{\bullet}, \mathrm{S}-18 \mathrm{mg} / \mathrm{kg}$ and radiation 5 Gy. Each data point represents the mean volume of 6-9 tumors. Bars, SE.

the normalized growth delay (NGD), defined as the time for tumors treated with both TS- 1 and radiation to grow to 4 times their pretreatment volume minus the time in days for tumors treated with TS-1 alone to reach the same volume. The enhancement factor (EF) was obtained by dividing NGD with the AGD radiation alone. Groups consisted of 8 to 10 mice each.

Thymidylate synthase (TS) immunohistochemical analysis. Tumor xenografts treated with vehicle, $8 \mathrm{mg} / \mathrm{kg} \mathrm{S}-1$ for 14 days, $5 \mathrm{~Gy}$, or the combination of the two agents were assessed immunohistochemically for thymidylate synthase (TS) at the end of the treatment with S-1. Using a method described by Miyamoto et al (25), the immunoreactivity of TS was examined using an anti-recombinant human TS monoclonal antibody (RTSMA1) (26), kindly supplied by Dr Masakazu Fukushima (Taiho Pharmaceutical Co., Ltd., Tokushima, Japan). All immunohistochemical examinations were performed on tissue sections of formalin-fixed, paraffin-embedded specimens from untreated and treated tumors. Serial $3-\mu \mathrm{m}$-thick slices were cut, deparaffinized in xylene, dehydrated with graded ethanol, and then immersed in methanol with $0.3 \%$ hydrogen peroxidase for $20 \mathrm{~min}$ to inhibit endogenous peroxidase activity. After washing in distilled water, the sections were placed in a $10 \mathrm{mM}$ citrate buffer solution ( $\mathrm{pH}$ 6.0). For immunohistochemistry, the slides were heated twice at $95^{\circ} \mathrm{C}$ for $10 \mathrm{~min}$ in a microwave oven and cooled for $30 \mathrm{~min}$ at room temperature. After washing in phosphate-buffered saline (PBS), non-specific binding was blocked by preincubation with $2 \%$ normal swine serum in PBS (blocking buffer) for $60 \mathrm{~min}$ at room temperature. All sections were incubated overnight at $4^{\circ} \mathrm{C}$ with the primary antibodies in blocking buffer at the following concentrations: RTSMA1 1:500 $(2 \mu \mathrm{g} / \mathrm{ml})$. After washing five times in PBS with $0.1 \%$ Tween-20 (washing buffer), the slides were incubated with biotinylated second anti-mouse (TS) antibody, diluted 1:200 with blocking buffer for $30 \mathrm{~min}$. After five washes with washing buffer, the sections were incubated with $\mathrm{ABC}$ reagent (Vector Laboratories Buringame, $\mathrm{CA}$ ), and a color reaction was developed using 2\% 3-3'- diaminobenzidine in $50 \mathrm{mM}$ Tris buffer ( $\mathrm{pH}$ 7.6) containing $0.3 \%$ hydrogen peroxide, for 5 to $10 \mathrm{~min}$. The sections were counterstained with Meyer's hematoxylin. In the negative controls, the primary antibody solution was replaced by the blocking buffer.

Evaluation of immunostaining. We randomly picked 10 areas in each slide and determined the immunostaining positivity based on a subjective estimation of intensity ( 0 to 4$)$ in each area. $0,<10 \%$ of tumor staining positive; $1,10-30 \%$ of tumor staining positive; $2,30-50 \%$ of tumor staining positive; 3,50 $70 \%$ of tumor staining positive; $4,>70 \%$ of tumor staining positive. Intensity levels 0 to 2 were considered negative, whereas 2 to 4 staining intensity was considered positive.

\section{Results}

Effect of TS-1 on radioresponse of DLD-1 and DLD-1/FU xenografts. Tumor xenografts, generated in the right hind thighs of nude mice by either DLD-1 or DLD-1/FU cells, were $80-100 \mathrm{~mm}^{3}$ when the treatments were initiated. Mice received a single dose of 5 Gy locally to the tumor, $8 \mathrm{mg} / \mathrm{kg}$ S-1 orally daily for 14 days, or both treatments. When the two agents were combined, the first dose of S-1 was administered several hours before tumor irradiation. The relative increase in tumor volume from the start of the treatments is shown in Fig. 1A for the DLD-1 tumor and in Fig. 1B for the DLD-1/ FU tumor. S-1, at a dose used here, was more effective than 5 Gy in slowing the growth of the DLD-1 tumor. The combined S-1 plus radiation treatment resulted in greater growth delay than that after the individual treatments, but the effect did not exceed the additive effect by the individual treatments. In comparison to the DLD-1 tumor, the DLD$1 / \mathrm{FU}$ tumor responded less well to both radiation and S-1 (Fig. 1B). However, when the two agents were combined, the effect on tumor growth delay was greater than the sum of the effects produced by the individual treatments. To reach $4 x$ the pretreatment volume (Fig. 2), tumors in untreated mice needed $8.7 \pm 2.9$ days, irradiated tumors $10.7 \pm 1.9$ days (AGD $=2.0$ days), tumors in mice treated with TS-1 $12 \pm 2.2$ 


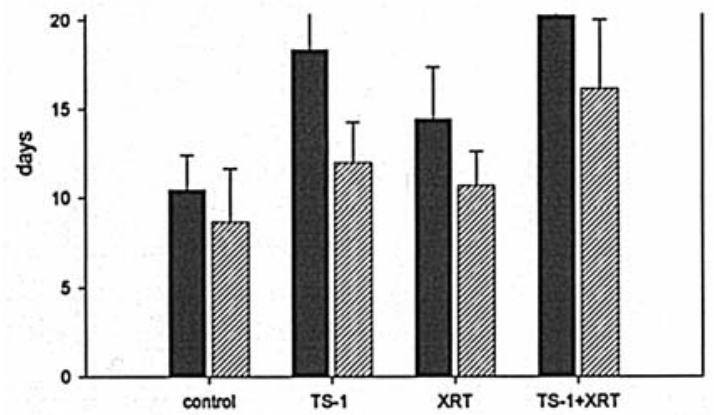

Figure 2. Effect of S-1 on the time taken to increase tumor size 1 to 4 times. Black bar, DLD-1; hatched bar, DLD-1/FU. Each data point represents the mean data of 6-9 tumors. Bars, SE.

days $(\mathrm{AGD}=3.3$ days), and tumors in mice treated with both of these agents needed $16.1 \pm 3.8$ days $(\mathrm{AGD}=7.4$ days; $\mathrm{NGD}=4.1$ days). This increase in tumor radioresponse was by a factor of 2.1 , obtained by dividing NGD of the combined treatment (4.1 days) with the AGD of 2 after radiation alone. This implies that the effect of S-1 plus radiation was synergistic (Table I).

Effect of 5-FU on in vitro cell radiosensitivity. To determine whether the S-1 metabolite, which is 5-FU, affects radiosensitivity of DLD-1 and DLD-1/FU cells, we determined the in vitro clonogenic cell survival. DLD-1 cells were exposed to $5.8 \mu \mathrm{M}$ and DLD-1/FU cells to $350 \mu \mathrm{M} \mathrm{S}-1$ for two days and then treated with 2 to 6 Gy of X-ray and plated to determine colony formation. After 10 days the DLD- 1 cell colonies or after 14 days the DLD-1/FU cell colonies were counted and the survival curves constructed (Fig. 3). Radiation caused a dose-dependent reduction in the cell survival of both cell lines, with DLD-1/FU being more radio-resistant than DLD-1. Treatment with 5-FU only slightly increased the radiation-induced cell killing of DLD-1 cells, but it was strongly effective in increasing the radiation-induced cell killing of DLD-1/FU cells. The enhancement of radiation response of DLD-1/FU cells at the 0.1 cell survival level by a factor of 1.9. Treatment with 5-FU also changed the shape of the radiation cell survival curve by almost completely removing the 'shoulder' region, suggesting that 5-FU may have reduced the ability of tumor cells to repair sublethal radiation damage.

Expression of thymidylate synthase in xenografts. Because thymidylate synthase is an enzyme involved in tumor resistance to both 5-FU (27-29) and radiation (29), it was important to determine whether the levels of this enzyme were affected in DLD-1/FU tumor xenografts treated with $\mathrm{S}-1$, radiation or the combination of the two agents. The doses and schedules of the agents were the same as in the in vivo experiment described above. The expression of protein levels of thymidylate synthase was assessed using immunohistochemistry at the end of the chemotherapy treatment, i.e. 14 days after treatment initiation, respectively. Fig. 4 illustrates the thymidylate synthase immunohistochemistry staining of tumor xenografts, untreated or treated with S-1, radiation or both with $\mathrm{x} 200$ magnifications. Table II shows the quantitative values for each group. These values were $3.58 \pm 0.6$ for untreated tumors, $3.25 \pm 0.7$ for tumors treated with $\mathrm{S}-1,3.44 \pm 0.7$ for tumors treated with radiation and $1.52 \pm 0.6$ for tumors treated with both $S-1$ and radiation. Thus, these results show that neither S-1 nor 5 Gy given as single treatments significantly affected the expression of thymidylate synthase, but when combined they strongly down-regulated the expression of this enzyme.

Table I. Enhancement factor.

\begin{tabular}{|c|c|c|c|c|}
\hline & Days of 1-4 folds & Absolute growth delay & Normalized growth delay & $\mathrm{EF}$ \\
\hline \multicolumn{5}{|l|}{ DLD-1 } \\
\hline control & 10,45708 & - & - & - \\
\hline TS-1 $8 \mathrm{mg} / \mathrm{kg}$ & 18,3139 & 7,85682 & - & - \\
\hline XRT 5 Gy & 14,4361 & 3,97902 & - & - \\
\hline TS-1+XRT & 20,2407 & 9,78362 & 1,9268 & 0,48424 \\
\hline \multicolumn{5}{|l|}{ DLD-1/FU } \\
\hline control & 8,708717 & - & - & - \\
\hline $\mathrm{TS}-18 \mathrm{mg} / \mathrm{kg}$ & 12,0044 & 3,295683 & - & - \\
\hline XRT 5 Gy & 10,7099 & 2,001183 & - & - \\
\hline TS-1+XRT 5 Gy & 16,1345 & 7,425783 & 4,1301 & 2,063829 \\
\hline
\end{tabular}




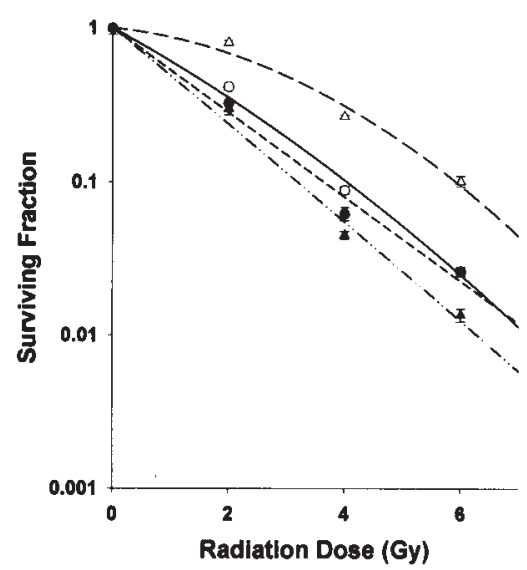

Figure 3. Effect of 5-FU on radiosensitivity of DLD-1 cells and DLD-1/FU cells in vitro. Cells were treated with 5-FU (5.8 $\mu \mathrm{M}$ for DLD-1, $350 \mu \mathrm{M}$ for DLD-1/FU) for 2 days before radiation. O, DLD-1 control; $\bullet$, DLD1/normal, 5-FU 5.8 $\mu \mathrm{M} 2$ days; $\square$, DLD-1/FU control; $\mathbf{\nabla}$, DLD-1/FU, 5-FU $350 \mu \mathrm{M} 14$ days. Values shown are the means \pm SE for three independent experiments.
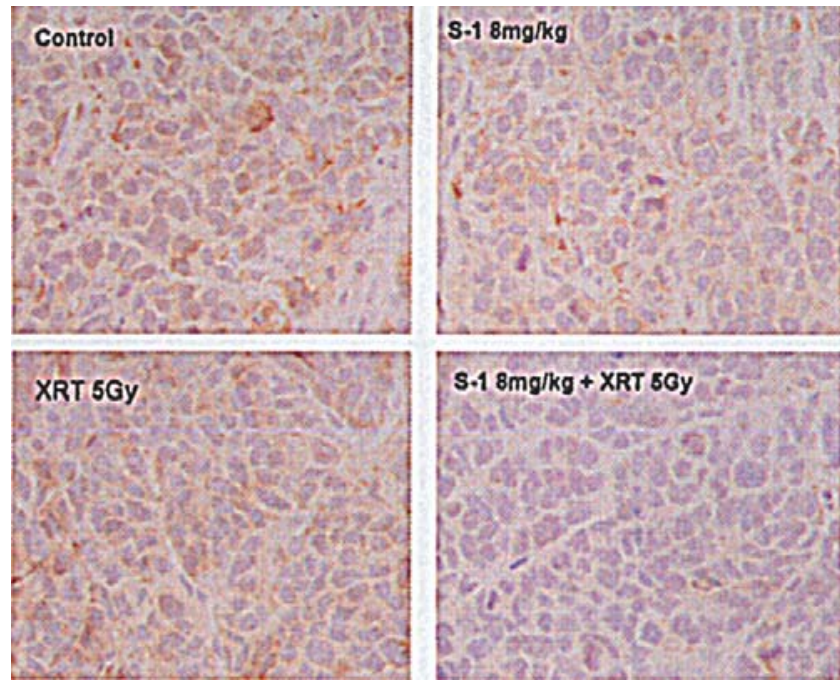

Figure 4. Effect of S-1 on the expression of thymidylate synthase. The thymidylate synthase immunohistochemistry staining of DLD-1/FU tumor xenografts. Upper left, no treatment; lower left, treated with radiation $5 \mathrm{~Gy}$; upper right, treated with S-1 $8 \mathrm{mg} / \mathrm{kg}$; lower right, treated with S-1 $8 \mathrm{mg} / \mathrm{kg}$ plus radiation $5 \mathrm{~Gy}$. TS staining is seen in the cytoplasm area. Original magnification $\mathrm{x} 200$.

\section{Discussion}

Chemotherapy using 5-FU, or 5-FU-based chemotherapy, in combination with radiotherapy has been a common treatment for many types of human cancer for several decades. This combination treatment improved local tumor control and patient survival rates in many cancers such as head and neck, pancreatic, cervical, esophageal and gastric cancer. It also resulted in improved organ preservation with a good functional outcome in a number of anatomic sites including head and neck and the rectum where it improved sphincter preservation. A variety of administration schedules of 5-FU and radiotherapy has been developed to optimize the antitumor effectiveness and minimize normal tissue toxicity.
Table II. Evaluation of immunohistochemistry of TS.

\begin{tabular}{lll}
\hline & \multicolumn{2}{c}{ Evaluation of IHC } \\
\cline { 2 - 3 } & \multicolumn{2}{c}{ Two weeks after initial treatment } \\
\hline Control & $3.58 \pm 0.577$ & (TS positive) \\
S-1 $8 \mathrm{mg} / \mathrm{kg}$ & $3.25 \pm 0.672$ & (TS positive) \\
Radiation 5 Gy & $3.44 \pm 0.647$ & (TS positive) \\
S-1+radiation & $1.52 \pm 0.624$ & (TS positive)
\end{tabular}

Evauation value was determined by the immunostaining positivity based on a subjective estimation of intensity ( 0 to 4 ) in each area. 0 , $<10 \%$ of tumor staining positive; $1,10-30 \%$ of tumor staining positive; $2,30-50 \%$ of tumor staining positive; $3,50-70 \%$ of tumor staining positive; $4,>70 \%$ of tumor staining positive. Intensity levels 0 to 2 were considered negative, whereas 2 to 4 staining intensity was considered positive.

Although there is no universal schedule, a prolonged continuous infusion of 5-FU has been shown to be superior to dose bolus injection both in terms of tumor response and more acceptable toxicity profile (6). However, poor bioavailability of 5-FU if administered orally, inconvenience with protracted i.v. administration of 5-FU, and a high rate of normal toxicity especially when combined with radiotherapy are issues that have stimulated research on developing oral fluoropyrimidines that would be more effective against tumors and less toxic for normal tissues, either when used alone or in combination with radiotherapy. S-1 was developed with the aim to meet such expectations.

Our present study tested whether S-1 can improve the antitumor efficacy of ionizing radiation, and the effect was assessed using colorectal carcinoma cells, either sensitive (DLD-1 cells) or resistant (DLD-1/FU cells) to 5-FU. The results showed that $\mathrm{S}-1$ increased the in vivo radioresponse of tumor xenografts generated by these cells, and that the active component 5-FU enhanced the in vitro sensitivity of these cells. However, these effects both in vitro and in vivo greatly depended on the sensitivity of cells to 5-FU, with S-1 (in vivo) and 5-FU (in vitro) being strongly effective against DLD-1/FU cells resistant to 5-FU. As shown in Fig. 3, the 5-FU sensitive DLD-1 cells are also more sensitive to ionizing radiation than the 5-FU resistant DLD-1/FU cells. Interestingly, while the 5-FU sensitive cells showed only a slight increase in radiosensitivity when exposed to 5-FU, 5-FU resistant cells were highly radiosensitized, with the enhancement factor of 1.86 at the 0.1 level of cell survival.

S-1 was strongly effective when combined with radiation in the treatment of tumor xenografts. Similarly to the in vitro findings, S-1-induced potentiation of the tumor radioresponse differed between DLD-1 and DLD-1/FU tumors. The DLD-1 tumor was sensitive to the treatment with $\mathrm{S}-1$ only. The combined S-1 plus radiation treatment resulted in a greater antitumor efficacy when compared with the efficacy of the individual treatments, but the effects were less than additive. In contrast, although the DLD-1/FU tumor was less responsive to radiation and $\mathrm{S}-1$ as individual treatments, it responded 
more than additively when the two agents were combined (Fig. 1B). The enhancement factor was 2.1. Harada et al (22) recently reported that $\mathrm{S}-1$ given either $1 \mathrm{~h}$ before or $1 \mathrm{~h}$ after irradiation, given for 5 consecutive days, improved radioresponse of human head and neck carcinomas, notably B88 squamous cell carcinoma cells derived from a tongue lesion and HSG salivary gland carcinoma cells. These cells were also radiosensitized when treated in vitro with 5-FU. However, the magnitude of the increase in radioresponse both in vitro and in vivo was much higher for DLD-1/FU cells we used in the present study than for head and neck carcinoma cells reported by Harada et al (22). In that study, the radiosensitivity of $\mathrm{B} 88$ cells was increased by a factor of 1.45 , and that of HSG cells by a factor of 1.28.

The mechanisms that underlie the increase in the in vivo tumor response to radiation by a chemotherapeutic agent, including S-1, are more complex compared to the in vitro induction of radioenhancement. While in vitro studies demonstrate a direct interaction on cellular sensitivity, the in vivo tumor response depends on many more factors in addition to the direct effect of tumor cell radiosensitivity, including accelerated tumor cell regeneration, tumor angiogenesis and tumor hypoxia (1). An important observation of our study is that the combined S-1 and radiation treatment resulted in significant down-regulation of thymidylate synthase, an enzyme involved in tumor resistance to 5-FU (27-29), and radiation (29). Based on our in vitro data, it is likely that increased tumor cell sensitivity to radiation is an important component responsible for the increased radioresponse of in vivo tumors. A similar explanation was given for the S-1-induced increase in the radioresponse of human head and neck tumor xenografts, and mechanistically the observed effect was attributed to the induction of apoptosis (22). Because 5-FU constitutes the cytotoxic component of $\mathrm{S}-1$, it is reasonable to assume that both $\mathrm{S}-1$ and 5-FU have similar mechanisms that underlie their radiosensitizing properties. Earlier studies on 5-FU show that this agent enhances tumor cell radio-sensitivity through cell cycle effects and inhibition of the repair of radiation-induced DNA damage $(8,30,31)$. The involvement of this latter mechanism in the observed radioenhancement of DLD-1/FU cells (Fig. 2) is strongly suggested by the shape of the radiation-dose survival curve when the cells were exposed to 5-FU: almost complete loss of the shoulder region fn the cell survival curve.

In conclusion, our results show that treatment with S-1, oral fluoropyrimidine, of tumor DLD-1/FU xenografts derived from human colon carcinoma cells resistant to 5-FU enhances the response of these tumors to local tumor radiotherapy. This response was associated with the downregulation of thymidylate synthase, an enzyme involved in tumor resistance to 5-FU and radiation. In vitro tumor cell survival results showed that S-1 (its active component 5-FU) is a potent enhancer of the radiosensitivity of DLD-1/FU cells, which implies that in vivo potentiation of tumor radioresponse is at least partly due to direct interaction between $\mathrm{S}-1$ and radiation on tumor cells. Because of a low toxicity profile of S-1 on normal tissues, these data suggest that S-1 has a potential to improve the therapeutic gain when combined with radiotherapy for colon cancer resistant to 5-FU.

\section{References}

1. Milas L, Mason KA, Liao Z, et al: Chemoradiotherapy: emerging treatment improvement strategies. Head Neck 25: 152-167, 2003.

2. Herskovic A, Marts K, al-Sarraf M, et al: Combined chemotherapy and radiotherapy compared with radiotherapy alone in patients with cancer of the esophagus. New Engl J Med 326: 1593-1598, 1992.

3. Brizel DM, Albers ME, Fisher SR, et al: Hyperfractionated irradiation with or without concurrent chemotherapy for locally advanced head and neck cancer. New Engl J Med 338: 1798-1804, 1998.

4. Morris M, Eifel PJ, Lu J, et al: Pelvic radiation with concurrent chemotherapy compared with pelvic and para-aortic radiation for high-risk cervical cancer. New Engl J Med 340: 1137-1143, 1999.

5. Furuse K, Fukuoka M and Kawahara M: Phase III study of concurrent versus sequential thoracic radiotherapy in combination with mitomycin, vindesine, and cisplatin in unresectable stage III non-small-cell lung cancer. J Clin Oncol 17: 2692-2699, 1999.

6. Rich TA, Shepard RC and Mosley ST: Four decades of continuing innovation with fluorouracil: current and future approaches to fluorouracil chemoradiation therapy. J Clin Oncol 22: 2214-2232, 2004.

7. Brito RA, Medgyesy D, Zukowski TH, et al: Fluoropyrimidines: a critical evaluation. Oncology 57 (suppl 1): 2-8, 1999.

8. Byfield J: Useful interactions between 5-fluorouracil and radiation in man: 5-fluorouracil as a radiosensitizer. In: Antitumor Drug-Radiation Interactions. Hill BT BA (eds). CRC Press Inc., Boca Raton, FL, pp87-105, 1990.

9. Lokich JJ, Ahlgren JD, Gullo JJ, et al: A prospective randomized comparison of continuous infusion fluorouracil with a conventional bolus schedule in metastatic colorectal carcinoma: a Mid-Atlantic Oncology Program Study. J Clin Oncol 7: 425-432, 1989.

10. Shirasaka T, Shimamato Y, Ohshimo H, et al: Development of a novel form of an oral 5-fluorouracil derivative (S-1) directed to the potentiation of the tumor selective cytotoxicity of 5fluorouracil by two biochemical modulators. Anticancer Drugs 7: 548-557, 1996.

11. Giller SA, Zhuk RA and Lidak M: Analogs of pyrimidine nucleosides. I. N1-(alpha-furanidyl) derivatives of natural pyrimidine bases and their antimetabolities. Dokl Akad Nauk SSSR 176: 332-335, 1967.

12. Tatsumi K, Fukushima M, Shirasaka T and Fujii S: Inhibitory effects of pyrimidine, barbituric acid and pyridine derivatives on 5-fluorouracil degradation in rat liver extracts. Jpn J Cancer Res 78: 748-755, 1987.

13. Shirasaka T, Shimamoto Y and Fukushima M: Inhibition by oxonic acid of gastrointestinal toxicity of 5-fluorouracil without loss of its antitumor activity in rats. Cancer Res 53: 4004-4009, 1993.

14. Shirasaka T, Nakano K, Takechi T, et al: Antitumor activity of $1 \mathrm{M}$ tegafur-0.4 M 5-chloro-2,4-dihydroxypyridine-1 M potassium oxonate (S-1) against human colon carcinoma orthotopically implanted into nude rats. Cancer Res 56: 2602-2606, 1996.

15. Fukushima M, Shimamoto Y, Kato T, et al: Anticancer activity and toxicity of S-1, an oral combination of tegafur and two biochemical modulators, compared with continuous i.v. infusion of 5-fluorouracil. Anticancer Drugs 9: 817-823, 1998.

16. Sakata Y, Ohtsu A, Horikoshi N, et al: Late phase II study of novel oral fluoropyrimidine anticancer drug S-1 (1 M tegafur$0.4 \mathrm{M}$ gimestat-1 $\mathrm{M}$ otastat potassium) in advanced gastric cancer patients. Eur J Cancer 34: 1715-1720, 1998.

17. Koizumi W, Kurihara M, Nakano S, et al: Phase II study of S-1, a novel oral derivative of 5-fluorouracil, in advanced gastric cancer. For the S-1 Cooperative Gastric Cancer Study Group. Oncology 58: 191-197, 2000.

18. Van den Brande J, Schffski P, Schllens JH, et al: EORTC Early Clinical Studies Group early phase II trial of S-1 in patients with advanced or metastatic colorectal cancer. Br J Cancer 88: 648-653, 2003.

19. Taguchi T, Morimoto K, Horikoshi N, et al: An early phase II clinical study of S-1 in patients with breast cancer. S-1 Cooperative Study Group (Breast Cancer Working Group). Gan To Kagaku Ryoho 25: 1035-1043, 1998. 
20. Inuyama Y, Kida A, Tsukuda M, et al: Early phase II study of S-1 in patients with advanced head and neck cancer. S-1 Cooperative Study Group (Head and Neck Working Group). Gan To Kagaku Ryoho 25: 1151-1158, 1998.

21. Kawahara M, Furuse K, Sagawa Y, et al: Phase II study of S-1, a novel oral fluorouracil, in advanced non-small-cell lung cancer. Br J Cancer 85: 939-943, 2001.

22. Harada K, Kawaguchi S, Supriatno, et al: Combined effects of the oral fluoropyrimidine anticancer agent, S-1 and radiation on human oral cancer cells. Oral Oncology 40: 713-719, 2004.

23. Murakami Y, Kazuno H, Emura T, et al: Different mechanisms of acquired resistance to fluorinated pyrimidines in human colorectal cancer cells. Int J Oncol 17: 277-283, 2000

24. Fertil B and Malaise EP: Inherent cellular radiosensitivity as a basic concept for human tumor radiotherapy. Int J Radiat Biol 7: 621-629, 1981.

25. Miyamoto S, Boku N, Ohtsu A, et al: Clinical implications of immunoreactivity of thymidilate synthase and dihydropyrimidine dehydrogenase in gastric cancer treated with oral fluoropyrimidine (S-1). Int J Oncol 17: 653-658, 2000.

26. Okabe H, Koizumi K, Tsujimoto H, et al: Rpitope analysis and utility of monoclonal antibodies to native and recombinant human thymidilate synthase. Int J Mol Med 5: 133-138, 2000.
27. Yamachika T, Nakanishi H, Inada K, et al: A new prognostic factor for colorectal carcinoma, thymidylate synthase, and its therepeutic significance. Cancer 82: 70-77, 1998.

28. Metzger R, Leichman CG, Danenberg KD, et al: ERCC mRNA levels complement thymidylate synthase mRNA levels in predicting responce and survival for gastric cancer patients receiving combination cisplatin and fluorouracil chemotherapy. J Clin Oncol 16: 309-316, 1998.

29. Saga Y, Suzuki M, Mizukami H, et al: Enhanced expression of thymidylate synthase mediates resistanceof uterine cervical cancer cells to radiation. Oncology 63: 185-191, 2002.

30. Nakajima Y, Miyamoto T, Tanabe M, et al: Enhancement of mammalian cell killing by 5-Fluorouracil in combination with X-rays. Cancer Res 39: 3763-3767, 1979.

31. Berry RJ: Effects of some metabolic inhibitors on X-ray doseresponse curves for the survival of mammalian cells in vitro, and on early recovery between fractionated X-ray doses. Br J Radiol 39: 458-463, 1966. 